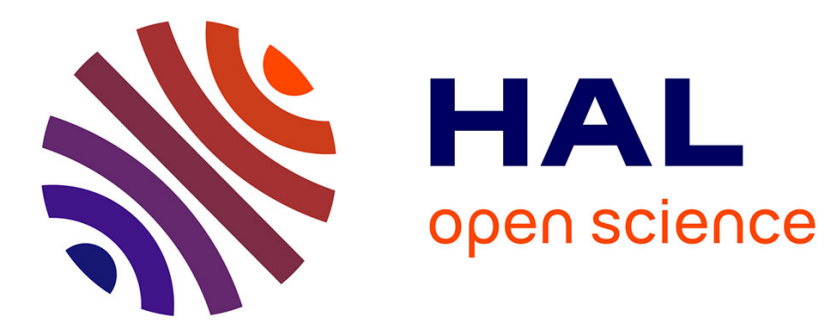

\title{
Acoustic streaming generated by two orthogonal standing waves propagating between two rigid walls
}

Alexander Doinikov, Pierre Thibault, Philippe Marmottant

\section{To cite this version:}

Alexander Doinikov, Pierre Thibault, Philippe Marmottant. Acoustic streaming generated by two orthogonal standing waves propagating between two rigid walls. Journal of the Acoustical Society of America, 2017, 141 (2), pp.1282-1289. 10.1121/1.4976088 . hal-01640077

\section{HAL Id: hal-01640077 \\ https://hal.science/hal-01640077}

Submitted on 20 Nov 2017

HAL is a multi-disciplinary open access archive for the deposit and dissemination of scientific research documents, whether they are published or not. The documents may come from teaching and research institutions in France or abroad, or from public or private research centers.
L'archive ouverte pluridisciplinaire HAL, est destinée au dépôt et à la diffusion de documents scientifiques de niveau recherche, publiés ou non, émanant des établissements d'enseignement et de recherche français ou étrangers, des laboratoires publics ou privés. 


\title{
Acoustic streaming generated by two orthogonal standing waves propagating between two rigid walls
}

\author{
Alexander A. Doinikov, ${ }^{a)}$ Pierre Thibault, and Philippe Marmottant \\ Laboratoire Interdisciplinaire de Physique, Unités Mixtes de Recherche 5588, Centre National de la \\ Recherche Scientifique, Université Grenoble-Alpes, Grenoble, F-38401, France
}

(Received 11 October 2016; revised 2 January 2017; accepted 27 January 2017; published online 28 February 2017)

\begin{abstract}
An analytical solution is derived for the acoustic streaming generated by two orthogonal standing waves in a fluid confined between two plane rigid walls. It is assumed that the standing waves have the same frequency but, in general, are out of phase. The main restriction is that the boundary layer thickness is much smaller than the acoustic wavelength. It is shown that the acoustic streaming gives rise to vortices in which fluid particles, when moving between the walls, are rotating about axes perpendicular to the walls. The location, the form, the sense of rotation of the vortices and the vortex strength are governed by the phase shift between the driving waves.
\end{abstract}

(C) 2017 Acoustical Society of America. [http://dx.doi.org/10.1121/1.4976088]

Pages: $1282-1289$

\section{INTRODUCTION}

The problem of acoustic streaming generated by a plane standing wave in a fluid channel confined between two plane rigid walls was first solved by Rayleigh. ${ }^{1}$ The vortex pattern outside the viscous boundary layer predicted by his theory is known as Rayleigh streaming. Various theoretical aspects of this problem have been analyzed by Westervelt, ${ }^{2}$ Nyborg, ${ }^{3,4}$ and Zarembo. ${ }^{5}$ The solutions obtained by Rayleigh, Westervelt, and Nyborg are valid for channels with a relatively large distance between the walls, in which the boundary layer thickness is negligible in comparison with the interwall distance. Zarembo's analysis is based on a solution for the sound field that is derived for a semi-infinite fluid in contact with a single wall, which excludes the application of his solution to channels with a finite distance between the walls. An analytical solution for acoustic streaming generated by a single standing wave in a channel with an arbitrary interwall distance was derived by Hamilton et al. ${ }^{6}$ They have shown that, as the distance between the channel walls is reduced, the streaming vortices inside the boundary layer increase in size relative to the Rayleigh streaming vortices outside the boundary layer, and for interwall distances less than about 10 times the boundary layer thickness, the Rayleigh vortices disappear and only the inner vortices exist.

In recent years, in the context of the advancement of acoustic microfluidics, ${ }^{7}$ there has been great interest in methods that allow one to generate stationary vortex flows in two-dimensional fluid-filled channels. This technology implies various applications, such as micromixing of fluids and the manipulation of microparticles suspended in a fluid. ${ }^{8-11}$ The present paper shows theoretically that two orthogonal standing waves, confined between two plane rigid walls, generate acoustic streaming that is of interest for the microfluidic applications mentioned above. Specifically, the streaming gives rise to rotational fluid motion in planes

${ }^{a)}$ Electronic mail: doinikov@hotmail.com parallel to the walls. As a result, fluid particles, when moving up and down between the walls, are rotating about axes perpendicular to the walls.

\section{THEORY}

Let us consider a viscous fluid confined between two plane rigid walls. The geometry of the problem is shown in Fig. 1. The walls are assumed to be at $z= \pm z_{0}$, which means that the plane $z=0$ is midway between them. Two orthogonal standing waves of the same frequency propagate along the $x$ and $y$ axes. From symmetry considerations, the $x$ and $y$ components of the fluid particle velocity must be even functions of $z$, while the $z$ component must be an odd function of $z$, such that it vanishes at $z=0$. All the velocity components are assumed to vanish at the walls. In microfluidics, it is commonly accepted to refer to the distance between the channel walls as channel height. We will follow this terminology.

\section{A. Model equations}

Hamilton et al. ${ }^{6,12}$ have developed an approximation approach for calculating the acoustic streaming generated by a single standing wave between two plane rigid walls, assuming that the viscous penetration depth is small compared to the acoustic wavelength. The generalization of their approach to the case of two orthogonal standing waves gives the following equations of fluid motion between the walls:

$$
\begin{aligned}
& \frac{\partial \rho}{\partial t}+\frac{\partial\left(\rho v_{i}\right)}{\partial x_{i}}=0, \\
& \frac{\partial\left(\rho v_{i}\right)}{\partial t}+\frac{\partial\left(\rho v_{i} v_{k}\right)}{\partial x_{k}}=-\frac{\partial p}{\partial x_{i}}+\eta \frac{\partial^{2} v_{i}}{\partial z^{2}}+f_{i}, \\
& \frac{\partial p}{\partial z}=0
\end{aligned}
$$

where $\rho$ is the fluid density, $v$ is the fluid velocity, $p$ is the fluid pressure, $\eta$ is the dynamic viscosity, and $f_{i}$ denotes a 


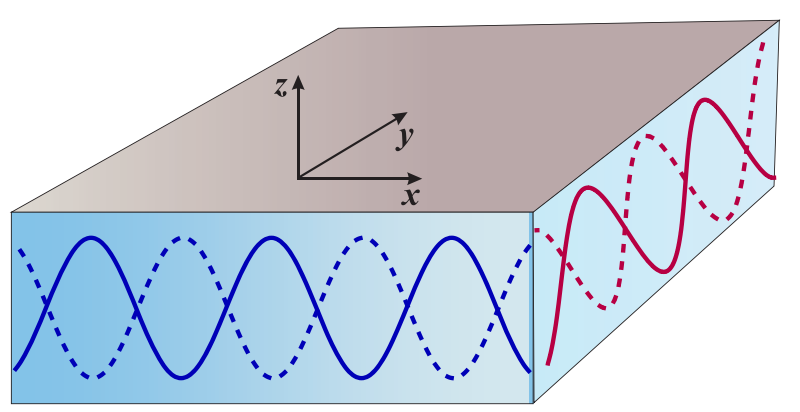

FIG. 1. (Color online) Geometry of the problem under consideration. Two orthogonal standing waves propagate between two plane rigid walls.

source (a body force per unit volume) that excites the standing waves in the fluid. The indices $i=1,2,3$ correspond to $x$, $y, z$ and summation over repeated indices is implied. The scale analysis that justifies Eqs. (1)-(3) is provided by Hamilton et al. ${ }^{6,12}$ (see also a paper by Waxler ${ }^{13}$ ) and therefore we do not dwell on it here. However, it should be mentioned that Hamilton et al. have demonstrated that their results are in agreement with the solutions derived by Rayleigh, ${ }^{1}$ Westervelt, ${ }^{2}$ and Nyborg. ${ }^{3}$ In what follows, we use Eqs. (1)-(3) to calculate the acoustic streaming in the case shown in Fig. 1.

\section{B. Linear solutions}

The linear fluid velocity can be represented as

$\boldsymbol{u}=\boldsymbol{u}_{x}+\boldsymbol{u}_{y}$,

where

$$
\boldsymbol{u}_{x}=u_{x x}(x, z, t) \boldsymbol{e}_{x}+u_{x z}(x, z, t) \boldsymbol{e}_{z}
$$

is the velocity produced by the standing wave propagating along the $x$ axis and

$$
\boldsymbol{u}_{y}=u_{y y}(y, z, t) \boldsymbol{e}_{y}+u_{y z}(y, z, t) \boldsymbol{e}_{z}
$$

is the velocity produced by the standing wave propagating along the $y$ axis. In view of linearity, $\boldsymbol{u}_{x}$ and $\boldsymbol{u}_{y}$ can be considered independently and hence, to get expressions for them, we can use the results obtained by Hamilton et al. ${ }^{6}$ for a single standing wave. These results, as applied to our case, yield

$$
\begin{aligned}
& u_{x x}=-u_{x 0}\left(1-\frac{\cosh \alpha x}{\cosh \alpha l}\right) F(z) e^{i \omega t}, \\
& u_{x z}=-u_{x 0} z_{0} \alpha \beta \frac{\sinh \alpha x}{\cosh \alpha l} G(z) e^{i \omega t}, \\
& u_{y y}=-u_{y 0}\left(1-\frac{\cosh \alpha y}{\cosh \alpha l}\right) F(z) e^{i \omega t}, \\
& u_{y z}=-u_{y 0} z_{0} \alpha \beta \frac{\sinh \alpha y}{\cosh \alpha l} G(z) e^{i \omega t} .
\end{aligned}
$$

Here,

$$
F(z)=1-\frac{\cosh k_{v} z}{\cosh k_{v} z_{0}}, \quad G(z)=\frac{z}{z_{0}}-\frac{\sinh k_{v} z}{\sinh k_{v} z_{0}},
$$

$$
\begin{aligned}
\alpha & =\frac{i \omega}{c \sqrt{1-\beta}}, \quad \beta=\frac{\tanh k_{v} z_{0}}{k_{v} z_{0}}, \quad k_{v}=\frac{1+i}{\delta_{v}}, \\
\delta_{v} & =\sqrt{\frac{2 \nu}{\omega}}
\end{aligned}
$$

$c$ is the speed of sound in the fluid, and $\nu$ is the kinematic viscosity. The quantities $u_{x 0}$ and $u_{y 0}$ can be regarded as the complex velocity amplitudes of the $x$ and $y$ standing waves, respectively. To introduce a phase shift $\phi$ between the $x$ and $y$ waves, it will suffice to set $u_{y 0}=u_{x 0} \exp (i \phi) . l$ is the distance between the center of the resonator and side walls along $x$ and $y$. It is assumed that the side walls are at the velocity nodes along both positive and negative directions of $x$ and $y$, so that $l=\lambda / 4+n \lambda / 2$, where $\lambda$ is the acoustic wavelength and $n=0,1,2, \ldots$.

\section{Equations of acoustic streaming}

We calculate the acoustic streaming in the approximation of an incompressible fluid. Averaging Eqs. (1) and (2) over time and substituting Eqs. (7)-(10), one obtains

$$
\begin{aligned}
\frac{\partial V_{x}}{\partial x}+\frac{\partial V_{y}}{\partial y}+\frac{\partial V_{z}}{\partial z}=0 & \\
\nu \frac{\partial^{2} V_{x}}{\partial z^{2}}= & \frac{\partial\left\langle u_{x x}^{2}\right\rangle}{\partial x}+\frac{\partial\left\langle u_{x x} u_{y y}\right\rangle}{\partial y}+\frac{\partial\left\langle u_{x x}\left(u_{x z}+u_{y z}\right)\right\rangle}{\partial z} \\
& +\frac{1}{\rho_{0}} \frac{\partial\langle p\rangle}{\partial x} \\
\nu \frac{\partial^{2} V_{y}}{\partial z^{2}}= & \frac{\partial\left\langle u_{y y} u_{x x}\right\rangle}{\partial x}+\frac{\partial\left\langle u_{y y}^{2}\right\rangle}{\partial y}+\frac{\partial\left\langle u_{y y}\left(u_{x z}+u_{y z}\right)\right\rangle}{\partial z} \\
& +\frac{1}{\rho_{0}} \frac{\partial\langle p\rangle}{\partial y},
\end{aligned}
$$

where $V_{x}=\left\langle v_{x}\right\rangle, V_{y}=\left\langle v_{y}\right\rangle, V_{z}=\left\langle v_{z}\right\rangle$ are the velocity components of the streaming, the angular brackets \langle\rangle denote the time average, and $\rho_{0}$ is the equilibrium fluid density. Note that $\langle p\rangle$ is a function of $x$ and $y$ only. The approximation approach developed by Hamilton et al. ${ }^{6,12}$ proposes that the $x$ and $y$ components of the streaming velocity, $V_{x}$ and $V_{y}$, should be calculated by Eqs. (14) and (15), respectively, and then the $z$ component, $V_{z}$, is calculated from Eq. (13).

The streaming velocity can be represented as

$$
\boldsymbol{V}=\boldsymbol{U}_{x}+\boldsymbol{U}_{y}+\boldsymbol{W}
$$

where

$$
\begin{aligned}
& \boldsymbol{U}_{x}=U_{x x}(x, z) \boldsymbol{e}_{x}+U_{x z}(x, z) \boldsymbol{e}_{z}, \\
& \boldsymbol{U}_{y}=U_{y y}(y, z) \boldsymbol{e}_{y}+U_{y z}(y, z) \boldsymbol{e}_{z}, \\
& \boldsymbol{W}=W_{x}(x, y, z) \boldsymbol{e}_{x}+W_{y}(x, y, z) \boldsymbol{e}_{y}+W_{z}(x, y, z) \boldsymbol{e}_{z} .
\end{aligned}
$$

The terms $\boldsymbol{U}_{x}$ and $\boldsymbol{U}_{y}$ describe the streaming velocities produced by the $x$ and $y$ waves, respectively, as if the second wave were absent. The term $\boldsymbol{W}$ stands for cross terms that result from the interaction of the $x$ and $y$ waves. It will be 
shown below that it is these cross terms that give rise to the fluid rotation in the $x y$ planes.

Substitution of Eqs. (16)-(18) into Eqs. (13)-(15) gives the following equations:

$$
\begin{aligned}
& \frac{\partial U_{x x}}{\partial x}+\frac{\partial U_{x z}}{\partial z}=0, \quad \frac{\partial U_{y y}}{\partial y}+\frac{\partial U_{y z}}{\partial z}=0 \\
& \nu \frac{\partial^{2} U_{x x}}{\partial z^{2}}=\frac{\partial\left\langle u_{x x}^{2}\right\rangle}{\partial x}+\frac{\partial\left\langle u_{x x} u_{x z}\right\rangle}{\partial z}+\frac{1}{\rho_{0}} \frac{d P_{x}(x)}{d x}, \\
& \nu \frac{\partial^{2} U_{y y}}{\partial z^{2}}=\frac{\partial\left\langle u_{y y}^{2}\right\rangle}{\partial y}+\frac{\partial\left\langle u_{y y} u_{y z}\right\rangle}{\partial z}+\frac{1}{\rho_{0}} \frac{d P_{y}(y)}{d y} \\
& \frac{\partial W_{x}}{\partial x}+\frac{\partial W_{y}}{\partial y}+\frac{\partial W_{z}}{\partial z}=0 \\
& \nu \frac{\partial^{2} W_{x}}{\partial z^{2}}=\frac{\partial\left\langle u_{x x} u_{y y}\right\rangle}{\partial y}+\frac{\partial\left\langle u_{x x} u_{y z}\right\rangle}{\partial z}+\frac{1}{\rho_{0}} \frac{\partial\left(\langle p\rangle-P_{x}\right)}{\partial x}, \\
& \nu \frac{\partial^{2} W_{y}}{\partial z^{2}}=\frac{\partial\left\langle u_{y y} u_{x x}\right\rangle}{\partial x}+\frac{\partial\left\langle u_{y y} u_{x z}\right\rangle}{\partial z}+\frac{1}{\rho_{0}} \frac{\partial\left(\langle p\rangle-P_{y}\right)}{\partial y},
\end{aligned}
$$

where $P_{x}\left(P_{y}\right)$ denotes a part of $\langle p\rangle$ that is generated if the $y$ $(x)$ wave vanishes.

An equation identical to Eqs. (20) and (21) has already been solved by Hamilton et al. ${ }^{6}$ using linear solutions identical to Eqs. (7)-(10). That is Eq. (37) in their paper. Therefore, expressions for $\boldsymbol{U}_{x}$ and $\boldsymbol{U}_{y}$ can be merely written by analogy. These expressions are given in the Appendix.

Two integrations of Eqs. (23) and (24) with respect to $z$ yield

$$
\begin{aligned}
W_{x}= & A_{x}(x, y)+\frac{z^{2}}{2 \eta} B_{x}(x, y)-\frac{1}{\omega} \operatorname{Re}\left\{\alpha u_{x 0}^{*} u_{y 0}\left(1-\frac{\cosh \alpha^{*} x}{\cosh \alpha^{*} l}\right)\right. \\
& \left.\times \frac{\sinh \alpha y}{\cosh \alpha l}\left[D_{1}(z)-D_{2}(z)\right]\right\}, \\
W_{y}= & A_{y}(x, y)+\frac{z^{2}}{2 \eta} B_{y}(x, y)-\frac{1}{\omega} \operatorname{Re}\left\{\alpha^{*} u_{x 0}^{*} u_{y 0}\left(1-\frac{\cosh \alpha y}{\cosh \alpha l}\right)\right. \\
& \left.\times \frac{\sinh \alpha^{*} x}{\cosh \alpha^{*} l}\left[D_{1}(z)-D_{2}^{*}(z)\right]\right\},
\end{aligned}
$$

where the asterisk indicates the complex conjugate, $A_{x}$ and $A_{y}$ are unknown functions to be determined by boundary conditions, and $B_{x}, B_{y}, D_{1}$, and $D_{2}$ are defined as

$$
\begin{aligned}
B_{x}(x, y)= & \frac{\partial\left(\langle p\rangle-P_{x}\right)}{\partial x}, \quad B_{y}(x, y)=\frac{\partial\left(\langle p\rangle-P_{y}\right)}{\partial y}, \\
D_{1}(z)= & \frac{1}{\delta_{v}^{2}} \iint F(z) F^{*}(z) d z^{2} \\
= & \frac{z^{2}}{2 \delta_{v}^{2}}+\frac{\cosh \left(2 z / \delta_{v}\right)-\cos \left(2 z / \delta_{v}\right)}{8\left|\cosh k_{v} z_{0}\right|^{2}} \\
& -\operatorname{Im}\left\{\frac{\cosh k_{v} z}{\cosh k_{v} z_{0}}\right\}
\end{aligned}
$$

$$
\begin{aligned}
D_{2}(z)= & \frac{\beta z_{0}}{\delta_{v}^{2}} \int G(z) F^{*}(z) d z \\
= & \frac{\beta z^{2}}{2 \delta_{v}^{2}}+\frac{i \cosh k_{v} z}{2 \cosh k_{v} z_{0}}+\frac{i \beta\left(\cosh k_{v}^{*} z-k_{v}^{*} z \sinh k_{v}^{*} z\right)}{2 \cosh k_{v}^{*} z_{0}} \\
& +\frac{\cosh \left[\left(k_{v}+k_{v}^{*}\right) z\right]-i \cosh \left[\left(k_{v}-k_{v}^{*}\right) z\right]}{4(1+i)\left|\cosh k_{v} z_{0}\right|^{2}}
\end{aligned}
$$

The integration also produces terms linear in $z$ but they were dropped because $W_{x}$ and $W_{y}$ must be even functions of $z$.

From the boundary condition $W_{x}=W_{y}=0$ at $z= \pm z_{0}$, one obtains

$$
\begin{aligned}
A_{x}(x, y)= & \frac{1}{\omega} \operatorname{Re}\left\{\alpha u_{x 0}^{*} u_{y 0}\left(1-\frac{\cosh \alpha^{*} x}{\cosh \alpha^{*} l}\right) \frac{\sinh \alpha y}{\cosh \alpha l}\right. \\
& \left.\times\left[D_{1}\left(z_{0}\right)-D_{2}\left(z_{0}\right)\right]\right\}-\frac{z_{0}^{2}}{2 \eta} B_{x}(x, y), \\
A_{y}(x, y)= & \frac{1}{\omega} \operatorname{Re}\left\{\alpha^{*} u_{x 0}^{*} u_{y 0}\left(1-\frac{\cosh \alpha y}{\cosh \alpha l}\right) \frac{\sinh \alpha^{*} x}{\cosh \alpha^{*} l}\right. \\
& \left.\times\left[D_{1}\left(z_{0}\right)-D_{2}^{*}\left(z_{0}\right)\right]\right\}-\frac{z_{0}^{2}}{2 \eta} B_{y}(x, y) .
\end{aligned}
$$

To find $W_{z}$, we substitute Eqs. (25) and (26) into Eq. (22) and integrate with respect to $z$. This yields the following equation:

$$
\begin{aligned}
W_{z}= & \frac{\delta_{v}|\alpha|^{2} D_{3}(z)}{\omega|\cosh \alpha l|^{2}} \operatorname{Re}\left\{u_{x 0}^{*} u_{y 0} \sinh \alpha^{*} x \sinh \alpha y\right\} \\
& -\frac{z^{3}}{6 \eta}\left(\frac{\partial B_{x}}{\partial x}+\frac{\partial B_{y}}{\partial y}\right)-z\left(\frac{\partial A_{x}}{\partial x}+\frac{\partial A_{y}}{\partial y}\right),
\end{aligned}
$$

where

$$
\begin{aligned}
D_{3}(z)= & \frac{2}{\delta_{v}} \int\left[\operatorname{Re}\left\{D_{2}(z)\right\}-D_{1}(z)\right] d z \\
= & -\frac{z^{3}}{3 \delta_{v}^{3}}-\frac{\sinh \left(2 z / \delta_{v}\right)-\sin \left(2 z / \delta_{v}\right)}{8\left|\cosh k_{v} z_{0}\right|^{2}}+\operatorname{Re}\left\{\frac{\beta z^{3}}{3 \delta_{v}^{3}}\right. \\
& \left.+\frac{\sinh \left[\left(k_{v}+k_{v}^{*}\right) z\right]-\sinh \left[\left(k_{v}-k_{v}^{*}\right) z\right]}{4(1+i)\left|\cosh k_{v} z_{0}\right|^{2}}\right\} \\
& +\operatorname{Im}\left\{\frac{(1-i) \sinh k_{v} z}{2 \cosh k_{v} z_{0}}\right. \\
& \left.+\frac{\beta\left[\left(k_{v}^{*} z\right)^{2} \sinh k_{v}^{*} z+2 k_{v}^{*} z \cosh k_{v}^{*} z-2 \sinh k_{v}^{*} z\right]}{2(1-i) \cosh k_{v}^{*} z_{0}}\right\} .
\end{aligned}
$$

The integration also produces a term dependent on $x$ and $y$ but it was dropped because $W_{z}$ must be an odd function of $z$.

Substitution of Eq. (32) into the boundary condition $W_{z}=0$ at $z= \pm z_{0}$ and the use of Eqs. (30) and (31) yield

$$
\frac{\partial B_{x}}{\partial x}+\frac{\partial B_{y}}{\partial y}=\frac{3 \rho_{0}|\alpha|^{2} \delta_{v}^{2} \varepsilon}{\left.2|\cosh \alpha|^{2}\right|_{0} ^{2}} \operatorname{Re}\left\{u_{x 0}^{*} u_{y 0} \sinh \alpha^{*} x \sinh \alpha y\right\},
$$


where

$$
\varepsilon=2 \operatorname{Re}\left\{D_{2}\left(z_{0}\right)\right\}-2 D_{1}\left(z_{0}\right)-\frac{\delta_{v}}{z_{0}} D_{3}\left(z_{0}\right) .
$$

From Eq. (34), one finds

$$
\begin{aligned}
& B_{x}(x, y)=\frac{3 \rho_{0} \delta_{v}^{2} \varepsilon}{4|\cosh \alpha l|^{2} z_{0}^{2}} \operatorname{Re}\left\{\alpha u_{x 0}^{*} u_{y 0} \cosh \alpha^{*} x \sinh \alpha y\right\} \\
& B_{y}(x, y)=\frac{3 \rho_{0} \delta_{v}^{2} \varepsilon}{4|\cosh \alpha l|^{2} z_{0}^{2}} \operatorname{Re}\left\{\alpha^{*} u_{x 0}^{*} u_{y 0} \sinh \alpha^{*} x \cosh \alpha y\right\} .
\end{aligned}
$$

Substitution of Eqs. (30), (31), (36), and (37) into Eqs. (25), (26), and (32) yields the final expressions for the components of $\boldsymbol{W}$ :

$$
\begin{aligned}
W_{x}= & \frac{1}{4 \omega|\cosh \alpha l|^{2}} \operatorname{Re}\left\{\alpha u_{x 0}^{*} u_{y 0} \sinh \alpha y\right. \\
& \times\left[3 \varepsilon\left(\frac{z^{2}}{z_{0}^{2}}-1\right) \cosh \alpha^{*} x+4\left(\cosh \alpha^{*} x-\cosh \alpha^{*} l\right)\right. \\
& \left.\left.\times\left(D_{1}(z)-D_{1}\left(z_{0}\right)+D_{2}\left(z_{0}\right)-D_{2}(z)\right)\right]\right\} \\
W_{y}= & \frac{1}{4 \omega|\cosh \alpha l|^{2}} \operatorname{Re}\left\{\alpha^{*} u_{x 0}^{*} u_{y 0} \sinh \alpha^{*} x\right. \\
\times & {\left[3 \varepsilon\left(\frac{z^{2}}{z_{0}^{2}}-1\right) \cosh \alpha y+4(\cosh \alpha y-\cosh \alpha l)\right.} \\
& \left.\left.\times\left(D_{1}(z)-D_{1}\left(z_{0}\right)+D_{2}^{*}\left(z_{0}\right)-D_{2}^{*}(z)\right)\right]\right\} \\
W_{z}= & \frac{|\alpha|^{2} z_{0}}{2 \omega|\cosh \alpha l|^{2}}\left[\varepsilon\left(\frac{z}{z_{0}}-\frac{z^{3}}{z_{0}^{3}}\right)+\frac{2 \delta_{v}}{z_{0}}\left(D_{3}(z)\right.\right. \\
& \left.\left.-\frac{z}{z_{0}} D_{3}\left(z_{0}\right)\right)\right] \operatorname{Re}\left\{u_{x 0}^{*} u_{y 0} \sinh \alpha^{*} x \sinh \alpha y\right\}
\end{aligned}
$$

\section{Lagrangian streaming}

The Lagrangian streaming velocity $\boldsymbol{V}_{L}$ is calculated as a sum of the Eulerian streaming velocity $\boldsymbol{V}$ and the Stokes drift velocity $\boldsymbol{V}_{S}$. The latter is given by ${ }^{14}$

$$
\boldsymbol{V}_{S}=\left\langle\int \boldsymbol{u} d t \cdot \nabla \boldsymbol{u}\right\rangle=\left\langle\frac{1}{i \omega}(\boldsymbol{u} \cdot \nabla) \boldsymbol{u}\right\rangle .
$$

Substitution of Eqs. (7)-(10) into Eq. (41) yields

$$
\begin{aligned}
V_{S x} & =\frac{1}{2 \omega|\cosh \alpha l|^{2}} \operatorname{Im}\left\{\left|u_{x 0}\right|^{2} \alpha^{*} \sinh \alpha^{*} x(\cosh \alpha x\right. \\
& -\cosh \alpha l)|F(z)|^{2}-z_{0} \alpha \beta\left(\left|u_{x 0}\right|^{2} \sinh \alpha x\right. \\
& \left.\left.+u_{x 0}^{*} u_{y 0} \sinh \alpha y\right)\left(\cosh \alpha^{*} x-\cosh \alpha^{*} l\right) G(z)\left[F^{\prime}(z)\right]^{*}\right\},
\end{aligned}
$$

$$
\begin{aligned}
V_{S y}= & \frac{1}{2 \omega|\cosh \alpha l|^{2}} \operatorname{Im}\left\{\left|u_{y 0}\right|^{2} \alpha^{*} \sinh \alpha^{*} y(\cosh \alpha y\right. \\
& -\cosh \alpha l)|F(z)|^{2}-z_{0} \alpha \beta\left(u_{x 0} u_{y 0}^{*} \sinh \alpha x\right. \\
& \left.+\left|u_{y 0}\right|^{2} \sinh \alpha y\right)\left(\cosh \alpha^{*} y-\cosh \alpha^{*} l\right) \\
& \left.\times G(z)\left[F^{\prime}(z)\right]^{*}\right\},
\end{aligned}
$$

$$
\begin{aligned}
V_{S z} & =\frac{z_{0}}{2 \omega|\cosh \alpha l|^{2}} \operatorname{Im}\left\{z_{0}|\alpha \beta|^{2} \mid u_{x 0} \sinh \alpha x\right. \\
& +\left.u_{y 0} \sinh \alpha y\right|^{2} G(z)\left[G^{\prime}(z)\right]^{*}+\alpha^{* 2} \beta^{*} F(z) G^{*}(z) \\
& \times\left[\left|u_{x 0}\right|^{2} \cosh \alpha^{*} x(\cosh \alpha l-\cosh \alpha x)\right. \\
& \left.\left.+\left|u_{y 0}\right|^{2} \cosh \alpha^{*} y(\cosh \alpha l-\cosh \alpha y)\right]\right\}
\end{aligned}
$$

where the prime denotes the derivative with respect to $z$.

\section{NUMERICAL SIMULATIONS}

Simulations were made at the following values of the physical parameters: $\rho_{0}=1000 \mathrm{~kg} / \mathrm{m}^{3}, \eta=0.001 \mathrm{Pas}$, $c=1500 \mathrm{~m} / \mathrm{s}, z_{0}=25 \mu \mathrm{m}$ (channel width $h=2, z_{0}=50 \mu \mathrm{m}$ ), $l=5 \lambda / 4$, and $f=36 \mathrm{MHz}$. These parameters are typical of microfluidic devices. ${ }^{15}$

Figure 2 shows streamlines of the projected streaming field on $x y$ planes at $z=0$ and $z=-0.9 z_{0}$. Similar patterns are observed for other values of $z$ as well. The phase shift between the $x$ and $y$ waves is $\phi=\pi / 2$. As one can see, the fluid is rotating about axes perpendicular to the walls. For the frequency used, $\delta_{v}=0.094 \mu \mathrm{m}$ and $z_{0} / \delta_{v} \approx 266$, so the vortices in Fig. 2 are of Rayleigh (outer) type.

Figure 3 provides an example of the 3D trajectory of a fluid particle involved in the acoustic streaming. The fluid particle is rotating in the central vortex shown in Fig. 2. It is moving upstream from the bottom wall located at $z=-z_{0}$. When doing so, the particle is rotating with a variable rotation radius about the vertical axis with the coordinates $x, y=0$. The starting point of the trajectory, shown by the black point in Fig. 3, has the coordinates $\left(0,0.2 \lambda,-0.95 z_{0}\right)$. It should be noted that Fig. 3 depicts only a part of the trajectory. The extension of the trajectory (not presented here in order to avoid overloading the figure) shows that, after moving up, the fluid particle is moving down, then up again, and so forth. The simulation also shows that the pitch of the spiral changes in the process of the particle motion. In the case shown in Fig. 3, the pitch increases as the particle is going up. When the particle approaches the upper point of the trajectory, the pitch of the spiral decreases. When the particle is going down, the pitch increases again. The strength of the 

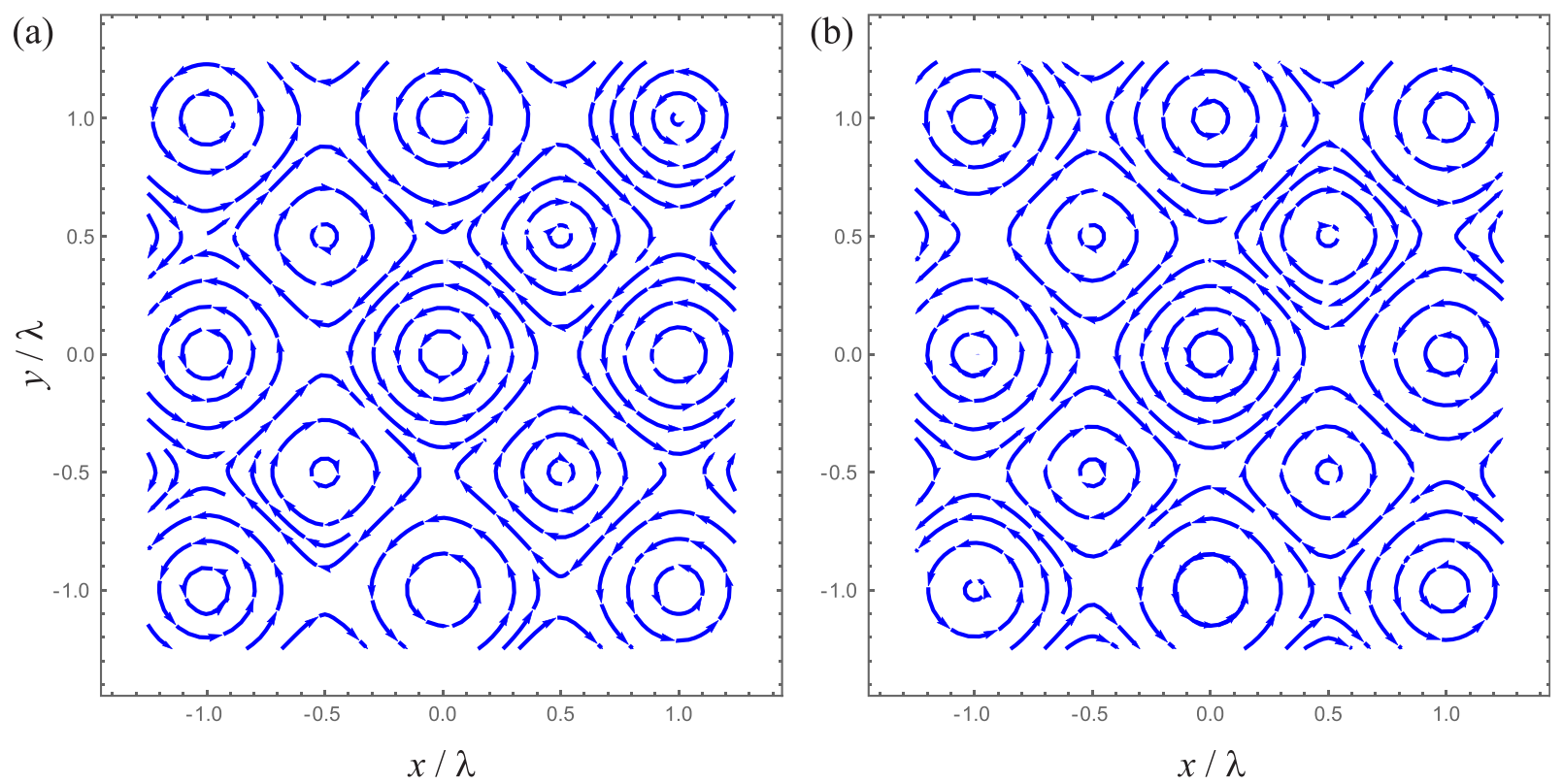

FIG. 2. (Color online) Streamlines of the projected streaming field on $x y$ planes at $z=(\mathrm{a}) 0$ and (b) $-0.9 z_{0}$. The calculation parameters are $f=36 \mathrm{MHz}$, $\phi=\pi / 2$, and $l=5 \lambda / 4$. The fluid is rotating about axes perpendicular to the walls.

driving acoustic field changes the velocity with which the fluid particle is moving along the trajectory but the shape of the trajectory remains invariable. The trajectory shape depends on the position of the starting point.

The calculation reveals that the rotation is caused by the cross terms of the streaming, $W_{x}$ and $W_{y}$. As an example, Fig. 4 shows what happens to Fig. 2(a) if the cross terms are eliminated from the calculation. As one can see, the vortices vanish.

Figures 5 shows the effect of the phase shift $\phi$ on the behavior of vortices. It depicts the streamlines of the

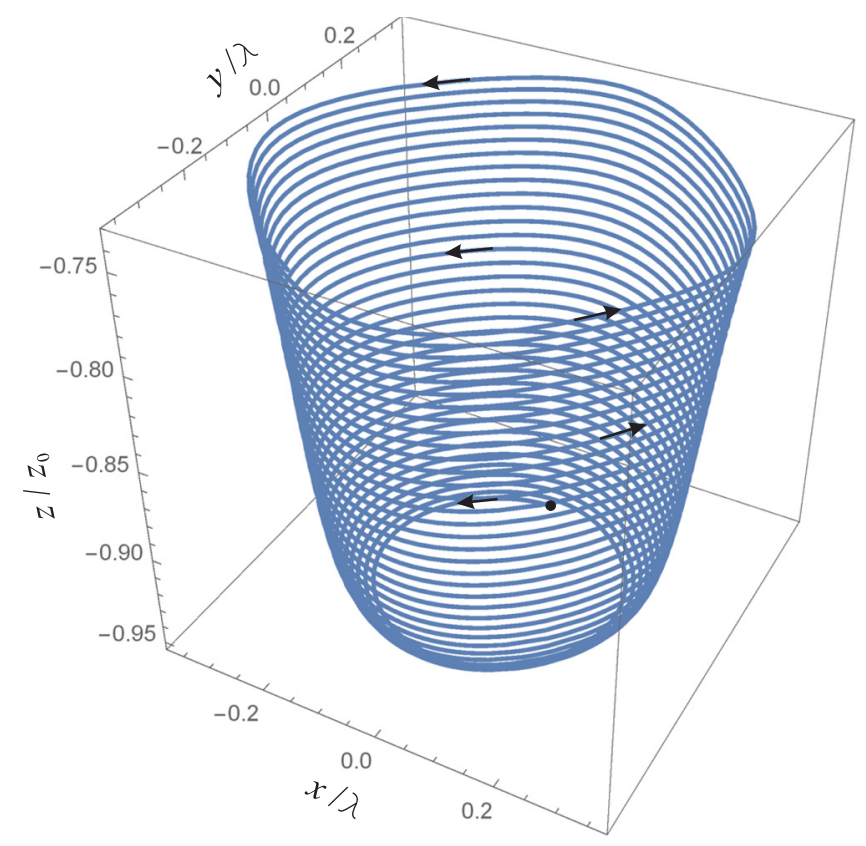

FIG. 3. (Color online) The 3D trajectory of a fluid particle rotating in the central vortex shown in Fig. 2. The starting point of the trajectory is $\left(0,0.2 \lambda,-0.95 z_{0}\right)$ (black point). The fluid particle is moving upstream from the bottom wall located at $z / z_{0}=-1$. projected streaming field on the $x y$ plane at $z=0$ for various values of the phase shift. As one can see, the phase shift changes the form, the location, and the sense of rotation of the vortices. The calculation also shows that $\phi$ in the range between $\pi$ and $2 \pi$ gives the same streaming patterns as $\phi$ between 0 and $\pi$ but with the opposite sense of rotation.

Finally, Fig. 6 demonstrates the effect of the phase shift on the vorticity of the streaming velocity, $\boldsymbol{\Omega}=\boldsymbol{\nabla} \times \boldsymbol{V}_{L}$. The vorticity is calculated in the center of the resonator at $x=y=z=0$; see the point in Fig. 5. The calculation reveals that at this point, the vorticity has only the $z$ component, $\Omega_{z}$, which is created by the streaming cross terms alone; see $\boldsymbol{W}$ in Eq. (16). The terms $\boldsymbol{U}_{x}$ and $\boldsymbol{U}_{y}$ are not able

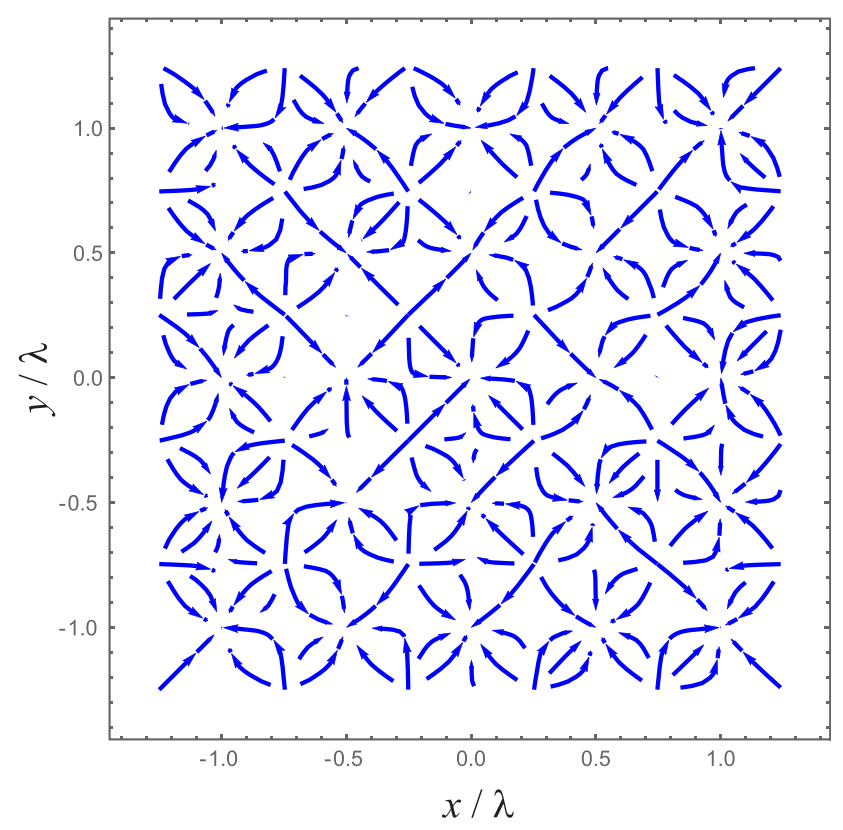

FIG. 4. (Color online) Example of calculation without the cross terms $W_{x}$ and $W_{y}$; cf. Fig. 2(a). Vortices vanish. 



FIG. 5. (Color online) Streamlines of the projected streaming field on the $x y$ plane at $z=0$ for various values of the phase shift $\phi$. The phase shift changes the form, the location, and the sense of rotation of vortices. 


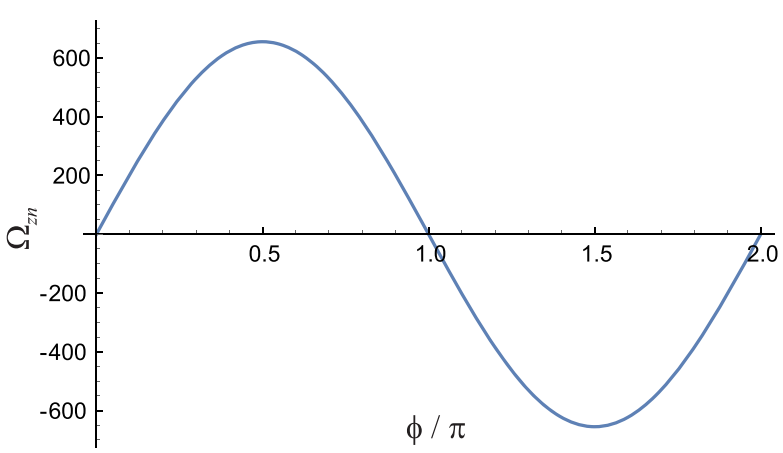

FIG. 6. (Color online) The normalized $z$ component of the vorticity of the streaming velocity at $x=y=z=0$ (see the point in Fig. 5) as a function of the phase shift $\phi$. The phase shift can increase or decrease the magnitude of the vorticity and change its sign.

to produce any rotation along the $z$ axis, and $\boldsymbol{\nabla} \times \boldsymbol{V}_{S}=0$ at $z=0$. Figure 6 depicts the normalized value of $\Omega_{z}$ as a function of the phase shift $\phi$. The normalization is performed as

$$
\Omega_{z n}=\frac{\omega|\cosh (\alpha l)|^{2}}{\left|\alpha^{2} u_{x 0} u_{y 0}\right|} \Omega_{z} .
$$

This normalization is suggested by the leading factor in Eq. (40). Figure 6 shows that the phase shift can increase or decrease the magnitude of the vorticity and change its sign. The peak values of the vorticity are reached at $\phi=\pi / 2$ and $3 \pi / 2$.

\section{CONCLUSION}

The present paper provides an analytical solution for the acoustic streaming generated by two orthogonal standing waves that propagate between two plane rigid walls. The derivation is based on the approximation proposed by Hamilton et al. ${ }^{6}$ The developed theory shows that the acoustic streaming gives rise to vortices in planes parallel to the walls. As a result, fluid particles, when moving up and down between the walls, are rotating with a variable rotation radius about axes perpendicular to the walls. The location, the form, the sense of rotation of the vortices and the vortex strength are governed by the phase shift between the driving waves. The obtained results are of interest for applications concerning the micromixing of fluids and the manipulation of microparticles suspended in a fluid.

\section{ACKNOWLEDGMENTS}

This work has received funding from the European Research Council under the European Union's Seventh Framework Programme (FP7/2007-2013)/ERC Grant agreement No. 614655 "Bubbleboost."

\section{APPENDIX: EXPRESSIONS FOR THE COMPONENTS OF $U_{x}$ AND $U_{y}$}

These expressions follow from the results obtained by Hamilton et al., ${ }^{6}$

$$
\begin{aligned}
U_{x x}(x, z)= & \frac{2\left|u_{x 0}\right|^{2}}{\omega l}\left[\frac{1}{z_{0}} A_{1}(x)+\frac{3 z^{2}}{z_{0}^{3}} B_{1}(x)+C_{1}(x, z)\right], \\
U_{x z}(x, z)= & -\frac{2\left|u_{x 0}\right|^{2}}{\omega l^{2}}\left[\frac{z}{z_{0}} A_{2}(x)+\frac{z^{3}}{z_{0}^{3}} B_{2}(x)\right. \\
& +\Psi_{2}(x, z)+\frac{z_{0}|\alpha l|^{2}}{4}\left|\frac{\left.\sinh \alpha x\right|^{2}}{\cosh \alpha l}\right|^{2} \\
& \left.\times \operatorname{Im}\left\{(1-\beta) \beta^{*}\left(\frac{z}{z_{0}}-\frac{\sinh k_{v}^{*} z}{\sinh k_{v}^{*} z_{0}}\right)\right\}\right] \\
U_{y y}(y, z)= & \frac{2\left|u_{y 0}\right|^{2}}{\omega l}\left[\frac{1}{z_{0}} A_{1}(y)+\frac{3 z^{2}}{z_{0}^{3}} B_{1}(y)+C_{1}(y, z)\right] \\
& \left.\times \operatorname{Im}\left\{(1-\beta) \beta^{*}\left(\frac{z}{z_{0}}-\frac{\sinh k_{v}^{*} z}{\sinh k_{v}^{*} z_{0}}\right)\right\}\right] \\
& +\Psi_{2}(y, z)+\frac{z_{0}|\alpha l|^{2}}{4}\left|\frac{\sinh \alpha y}{\cosh \alpha l}\right|^{2} \\
U_{y z}(y, z)= & \frac{2\left|u_{y 0}\right|^{2}}{\omega l^{2}}\left[\frac{z}{z_{0}} A_{2}(y)+\frac{z^{3}}{z_{0}^{3}} B_{2}(y)\right.
\end{aligned}
$$

where

$$
\begin{aligned}
A_{j}(x)= & -\frac{3}{2} \Psi_{j}\left(x, z_{0}\right)+\frac{z_{0}}{2} C_{j}\left(x, z_{0}\right), \\
B_{j}(x)= & \frac{1}{2} \Psi_{j}\left(x, z_{0}\right)-\frac{z_{0}}{2} C_{j}\left(x, z_{0}\right), \\
C_{j}(x, z)= & \operatorname{Re}\left\{G_{j}(x)\left[H_{1}(z)+i H_{2}(z)\right]\right\}, \\
\Psi_{j}(x, z)= & \delta_{v} \operatorname{Re}\left\{G_{j}(x)\left[H_{3}(z)+i H_{4}(z)\right]\right. \\
& \left.+\frac{i}{4}(1-\beta) G_{j}^{*}(x) H_{5}(z)\right\},
\end{aligned}
$$$$
G_{1}(x)=\alpha^{*} l \frac{\sinh \alpha^{*} x}{\cosh \alpha^{*} l}\left(\frac{\cosh \alpha x}{\cosh \alpha l}-1\right) \text {, }
$$$$
G_{2}(x)=\left(\alpha^{*} l\right)^{2} \frac{\cosh \alpha^{*} x}{\cosh \alpha^{*} l}\left(\frac{\cosh \alpha x}{\cosh \alpha l}-1\right)+\left|\alpha l \frac{\sinh \alpha x}{\cosh \alpha l}\right|^{2},
$$

$$
H_{1}(z)=\frac{\cosh \left(2 z / \delta_{v}\right)-\cos \left(2 z / \delta_{v}\right)}{8\left|\cosh k_{v} z_{0}\right|^{2}}-\operatorname{Im}\left\{\frac{\cosh k_{v} z}{\cosh k_{v} z_{0}}\right\} \text {, }
$$

$$
\begin{aligned}
H_{2}(z)= & \beta^{*} \frac{\cosh k_{v} z-k_{v} z \sinh k_{v} z}{4 \cosh k_{v} z_{0}}+\frac{\cosh k_{v}^{*} z}{4 \cosh k_{v}^{*} z_{0}} \\
& -\frac{\cosh \left(2 z / \delta_{v}\right)+i \cos \left(2 z / \delta_{v}\right)}{8 k_{v} \delta_{v}\left|\cosh k_{v} z_{0}\right|^{2}}
\end{aligned}
$$

$$
\begin{aligned}
H_{3}(z)= & \frac{\sinh \left(2 z / \delta_{v}\right)-\sin \left(2 z / \delta_{v}\right)}{16\left|\cosh k_{v} z_{0}\right|^{2}} \\
& -\operatorname{Im}\left\{\frac{\sinh k_{v} z}{k_{v} \delta_{v} \cosh k_{v} z_{0}}\right\},
\end{aligned}
$$




$$
\begin{aligned}
H_{4}(z)= & \beta^{*} \frac{2 \sinh k_{v} z-k_{v} z \cosh k_{v} z}{4 k_{v} \delta_{v} \cosh k_{v} z_{0}}+\frac{i \sinh k_{v}^{*} z}{4 k_{v} \delta_{v} \cosh k_{v}^{*} z_{0}} \\
& -\frac{\sinh \left(2 z / \delta_{v}\right)+i \sin \left(2 z / \delta_{v}\right)}{16 k_{v} \delta_{v}\left|\cosh k_{v} z_{0}\right|^{2}} \\
H_{5}(z)= & \frac{z}{\delta_{v}}-\frac{i \sinh k_{v}^{*} z}{k_{v} \delta_{v} \cosh k_{v}^{*} z_{0}} .
\end{aligned}
$$

${ }^{1}$ Lord Rayleigh, "On the circulation of air observed in Kundt's tubes, and on some allied acoustical problems," Philos. Trans. R. Soc. London 175, 1-21 (1884).

${ }^{2} \mathrm{P}$. J. Westervelt, "The theory of steady rotational flow generated by a sound field," J. Acoust. Soc. Am. 25, 60-67 (1953).

${ }^{3}$ W. L. Nyborg, "Acoustic streaming," in Physical Acoustics, edited by W. P. Mason (Academic, New York, 1965), Vol. 2B, Chap. 11, pp. 290-295.

${ }^{4}$ W. L. Nyborg, "Acoustic streaming," in Nonlinear Acoustics, edited by M. F. Hamilton and D. T. Blackstock (Academic, San Diego, 1998), Chap. 7, Sec. 3.3.

${ }^{5}$ L. K. Zarembo, "Acoustic streaming," in High-Intensity Ultrasonic Fields, edited by L. D. Rozenberg (Plenum, New York, 1971), Part III, pp. $156-164$.
${ }^{6}$ M. F. Hamilton, Yu. A. Ilinskii, and E. A. Zabolotskaya, "Acoustic streaming generated by standing waves in two-dimensional channels of arbitrary width," J. Acoust. Soc. Am. 113, 153-160 (2003).

${ }^{7} \mathrm{~J}$. Friend and L. Yeo, "Microscale acoustofluidics: Microfluidics driven via acoustics and ultrasonics," Rev. Mod. Phys. 83, 647-704 (2011).

${ }^{8}$ C. Wang, S. V. Jalikop, and S. Hilgenfeldt, "Size-sensitive sorting of microparticles through control of flow geometry," Appl. Phys. Lett. 99, 034101 (2011).

${ }^{9} \mathrm{~J}$. Lei, P. Glynne-Jones, and M. Hill, "Acoustic streaming in the transducer plane in ultrasonic particle manipulation devices," Lab Chip 13, 2133-2143 (2013).

${ }^{10} \mathrm{~J}$. Lei, M. Hill, and P. Glynne-Jones, "Numerical simulation of 3D boundary-driven acoustic streaming in microfluidic devices," Lab Chip 14, 532-541 (2014).

${ }^{11}$ N. Nama, R. Barnkob, Z. Mao, C. J. Kähler, F. Costanzo, and T. J. Huang, "Numerical study of acoustophoretic motion of particles in a PDMS microchannel driven by surface acoustic waves," Lab Chip 15, 2700-2709 (2015).

${ }^{12}$ M. F. Hamilton, Yu. A. Ilinskii, and E. A. Zabolotskaya, "Nonlinear twodimensional model for thermoacoustic engines," J. Acoust. Soc. Am. 111, 2076-2086 (2002).

${ }^{13} \mathrm{R}$. Waxler, "Stationary velocity and pressure gradients in a thermoacoustic stack," J. Acoust. Soc. Am. 109, 2739-2750 (2001).

${ }^{14}$ M. S. Longuet-Higgins, "Viscous streaming from an oscillating spherical bubble," Proc. R. Soc. London Ser. A 454, 725-742 (1998).

${ }^{15}$ S. B. Q. Tran, P. Marmottant, and P. Thibault, "Fast acoustic tweezers for the two-dimensional manipulation of individual particles in microfluidic channels," Appl. Phys. Lett. 101, 114103 (2012). 